\title{
Síndrome de dehiscencia del canal semicircular superior: Revisión
}

\section{Dehiscense of the semicircular superior channel Syndrome: Revision}

\author{
Jorge Caro $L^{1}$, Francisca Fernández $L^{2}$.
}

\begin{abstract}
RESUMEN
日 Síndrome de Dehiscencia del Canal Semicircular Superior (SDCSS) fue descrito en los últimos años en pacientes que presentaban vértigo y nistagmus inducido por presión asociado a un adelgazamiento de la pared del canal semicircular superior. $B$ diagnóstico del cuadro se basa en la clínica, sin embargo las técnicas de imágenes juegan un rol importante en la certificación del cuadro. $\theta$ tratamiento es esencialmente quirúrgico, dependiendo de la sintomatología. Creemos que es importante plantear este diagnóstico en todos los pacientes que consultan por vértigo, ya que podría ser una causa potencialmente tratable de su sintomatología
\end{abstract}

Palabras clave: conducto semicircular, vértigo, nistagmus.

\section{SUMMARY}

The dehiscense of the semicircular superior channel Syndrome (SDCSS) was described in the past years on patients who suffered from vertigo and nistagmus induced by pressure associated to a slimming of the superior semicircular canal wall. The diagnosis of the Picture is based on clinic, however the image techniques play an important role in the certification of the Picture. The treatment is essentially surgical, depending on the symptomatology. We believe it is important to consider this diagnosis in all patients who suffer vertigo, as it could be a potentially treatable cause of its symptomatology.

Key words: Semicircular canal, vertigo, nistagmus.

\section{INTRODUCCIÓN}

Por muchos años, los especialistas en otología han reconocido que, bajo ciertas condiciones, el sonido transmitido hacia el oído interno tendría la propie dad de activar el sistema vestibular o del equilibrio.

日 oído interno está compuesto por un componente coclear y un componente vestibular que en condiciones normales actúan de manera inde-

\footnotetext{
${ }^{1}$ UDA Otorrinolaringología, Facultad de Medicina, Pontificia Universidad Católica de Chile.

${ }^{2}$ Médico, Programa de Magíster en Ciencias Médicas, Mención Inmunología. Facultad de Medicina, Universidad de Chile. Residente Unidad Asociada, Otorrinolaringología Pontificia Universidad Católica de Chile.
} 
pendiente. Sin embargo, existen circunstancias en las que un sonido o presión pueden desencadenar sensación de vértigo y nistagmus en un paciente ${ }^{1}$.

En estudios realizados a principios del siglo veinte por Tullio ${ }^{2}$ y posteriormente por Huizinga ${ }^{3}$ se observó que la fenestración de los canales semicirculares en palomas, provocaba movimientos oculares y cefálicos en el mismo plano que el canal dañado. Además vieron que esta misma respuesta podía observarse frente a sonidos intensos, lo que se llamó fenómeno de Tullio, o al ejercer presión sobre el canal auditivo en ciertos estados patológicos en que estuviera afectada la integridad del hueso laberíntico, lo que recibió el nombre de signo de Hennebert ${ }^{4}$.

日 fenómeno de nistagmus y/o síntomas vestibulares evocados por sonido o presión ha sido descrito en pacientes con fístula perilinfática ${ }^{5}$, sordera congénita, enfermedad de Ménière de larga evolución ${ }^{1}$, enfermedad de Lyme, trauma cefálico y colesteatoma con erosión de los canales semicirculares ${ }^{3,4}$. En tales casos se planteaba que el mecanismo responsable de los síntomas sería la aparición de una "tercera ventana" debido a las alteraciones en el laberinto óseo.

En los años noventa fue descrita una nueva entidad que no podía ser explicada por ningún otro cuadro conocido. Los pacientes portadores de este trastorno presentaban uno o más de los siguientes síntomas: desequilibrio, pérdida auditiva, marcada hipersensibilidad a algunos sonidos de cierta frecuencia, intolerancia al movimiento y/o percepción de manera aumentada de algunos sonidos como los movimientos oculares o los latidos del corazón ${ }^{6}$.

Los estudios de imágenes realizados a estos pacientes revelaron un adelgazamiento del hueso que cubría al canal semicircular superior (CSS) del sistema vestibular, por lo que denominó a este cuadro Síndrome de Dehiscencia del Canal Semicircular Superior (SDCSS) 6 .

La causa originaria de esta condición aún no es completamente entendida a pesar de los numerosos estudios que ha motivado esta nueva entidad.

A continuación haremos una revisión sobre los distintos reportes que existen en la literatura sobre el tema y los últimos avances en cuanto a su diagnóstico y manejo.

\section{FISIOPATOLOGÍA}

Las primeras descripciones del cuadro fueron realizadas por Minor y colaboradores en 1998. Los investigadores identificaron un grupo de ocho pacientes que presentaba, en común, episodios de vértigo $\mathrm{y} / \mathrm{o}$ nistagmus desencadenados por sonidos de alta intensidad o por presión en el oído afectado, sin evidencia previa de enfermedades otológicas. En todos ellos se identificó una dehiscencia ósea del CSS en el oído correspondiente mediante tomografía axial computada Los pacientes estudiados presentaban movimientos oculares en el mismo eje del canal afectado 6 .

Minor planteó que la falta de cobertura ósea o dehiscencia en el CSS actuaría como una "tercera ventana" móvil a nivel de oído interno en forma adicional a las ventanas oval y redonda, lo que permitiría la transmisión de la vibración hacia el aparato vestibular, produciendo la sensación de vértigo 6,7 .

Se ha propuesto que el mecanismo involucrado en el SDCSS sería el movimiento de la ampolla del CSS debido a una complacencia aumentada del sistema endolinfático que sería generada por la dehiscencia.

En el estudio realizado en chinchillas, por Hirvonen y colaboradores, se analizaron las descargas aferentes del nervio vestibular en respuesta a los cambios de presión en el canal auditivo externo, antes y después de la fenestración del CSS. Observaron que después de realizada la fenestración todas las aferentes del CSS mostraban excitabilidad inducida por presión positiva, mientras que no se observó excitabilidad en las aferencias del canal semicircular horizontal. La respuesta observada frente a la presión desapare cía al ocluir con un sello rígido la fenestración ${ }^{8}$.

Los pacientes con una dehiscencia del CSS confirmada pueden presentar además síntomas auditivos, incluyendo sensibilidad disminuida a las frecuencias bajas (menores a $2 \mathrm{kHz}$ ) conducidas 
por vía aérea y una hipersensibilidad a frecuencias bajas (menores a $2 \mathrm{kHz}$ ) conducidas por vía ósea. La conjunción de ambos fenómenos se traduce en una hipoacusia de conducción de hasta 30 ó 60 $\mathrm{dB}^{7,9}$.

La hipótesis de esta tercera ventana ha sido usada también para explicar las alteraciones en la sensibilidad auditiva que presentan estos pacientes. La dehiscencia podría actuar como una vía de derivación de las ondas acústicas, disminuyendo la impedancia coclear y produciendo que una porción del volumen vestibular sea desplazado lejos de la cóclea y por lo tanto reduciendo el estímulo coclear $^{8}$. De la misma manera, la dehiscencia también produciría un aumento de la respuesta coclear al sonido transmitido por vía ósea, lo que explicaría los fenómenos de hipersensibilidad a sonidos transmitidos por esta vía que presentan algunos pacientes con SDCSS ${ }^{7}$.

Rozowski y colaboradores realizaron mediciones, con vibrometría láser, de la velocidad de vibración del estribo inducida por sonido en pacientes con SDCSS, que no habían sido sometidos previamente a cirugía. Observaron una tendencia a la hipermovilidad del estribo, lo que sería sugerente de una disminución en la impedancia coclear. Por otra parte, al estudiar las características de la endolinfa en chinchillas con dehiscencia del CSS frente a un estímulo acústico, se observó un aumento en la velocidad de la endolinfa hacia la dehiscencia, lo que apoyaría la hipótesis del desplazamiento de energía lejos de la cóclea debido a la fenestración ${ }^{10}$.

\section{ETIOLOGÍA}

La etiología de la dehiscencia en el CSS también ha sido motivo de numerosos estudios.

En el primer reporte de Minor ninguno de los pacientes estudiados tenía antecedentes de enfermedades otológicas previas, salvo episodios de otitis media aguda en la infancia 6 .

Carey y colaboradores publicaron un estudio en el que analizaron 1.000 huesos temporales de 596 adultos con el fin de determinar la incidencia, localización y etiología del SDCSS. Se identificó dehiscencia $(0,7 \%)$ o al menos adelgazamiento $(1,3 \%)$ del CSS en $2 \%$ de los individuos estudiados, siendo dos los sitios comprometidos con mayor frecuencia: el seno petroso y el punto más alto del CSS, donde se proyecta hacia la fosa media ${ }^{11}$.

No encontraron alteraciones locales en el hue so temporal que pudieran explicar la erosión óseay ninguno de los pacientes estudiados tenía historia previa de traumatismos craneanos. Además se observó que el adelgazamiento o dehiscencia era bilateral en la gran mayoría de los pacientes, por lo que los autores plantearon que alguna alteración

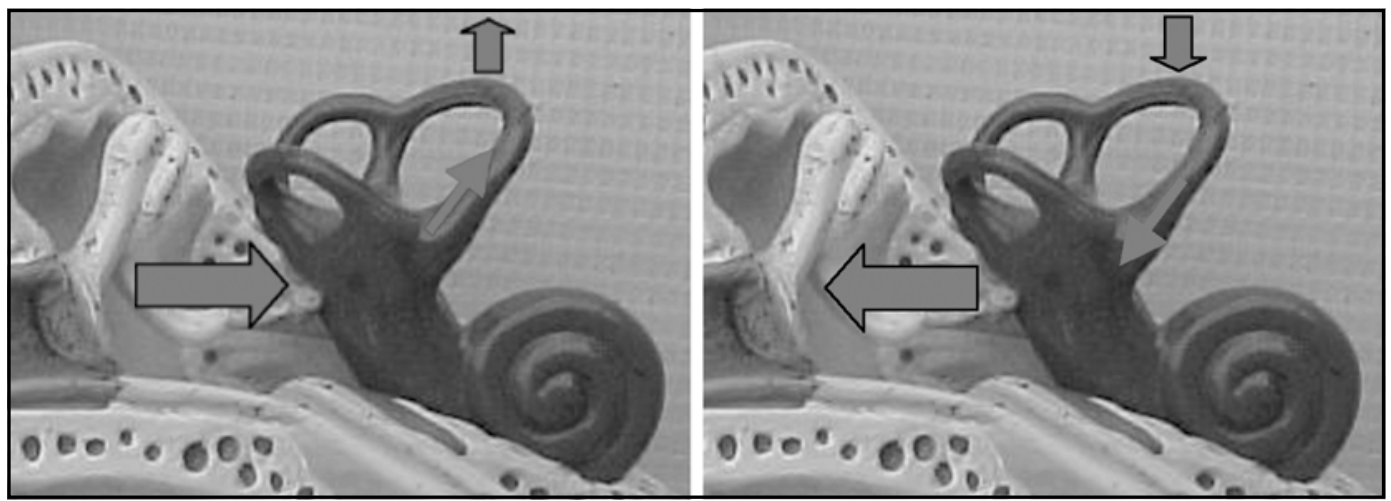

Figura 1. Efecto de tercera ventana propuesto en el SDCSS. Explicación en maqueta Dr. Jorge Caro. 
en etapas tempranas del desarrollo del individuo podría explicar estos hallazgos y que, probablemente, algún evento posterior, como un traumatismo o aumento de la presión intracraneana, podría acentuar esta condición y determinar la aparición de los síntomas ${ }^{11}$.

En otro estudio realizado por Williamson y colaboradores se observó una prevalencia de 9\% de SDCSS en estudios de tomografía axial computada, encontrándose un compromiso bilateral en nueve de los treinta casos diagnosticados como SDCSS. $\mathrm{\theta}$ promedio de edad de los pacientes estudiados fue de 45 años, con una distribución equivalente por sexo. Sin embargo, sólo un escaso porcentaje de los pacientes estudiados presentaban síntomas sugerentes de SDCSS $^{12}$.

\section{CLÍNICA}

Los pacientes portadores de este trastorno poseen características clínicas particulares, entre las que se incluyen: fenómeno de Tullio, vértigo u oscilopsias inducidos por sonidos fuertes o por presión (otoscopía neumática, maniobra de Valsalva) e hipoacusia, entre otras $6,7,9$. Es importante señalar que muchos de estos signos pueden no ser clínicamente evidentes al momento de la consulta, por lo que es importante realizar una anamnesis detallada y un examen físico acucioso ${ }^{13}$.

La presentación del SDCSS puede ser amplia y muy variable entre un paciente y otro; algunos pueden presentar síntomas vestibulares incapacitantes, pero con audición conservada, otros hipoacusia severa pero con función vestibular normal, o bien la combinación de ambos $^{6-8,13}$. Esta amplia variabilidad podría estar relacionada con el tamaño de la dehiscencia, ya que se ha observado que dehiscencias de menor diámetro no producen mayores cambios en los potenciales cocleares, mientras que las de mayor tamaño sí lo hacen ${ }^{14}$.

日 SDCSS también puede presentarse como una hipoacusia de conducción, que semeje una otoescle-rosis y podría explicar algunos casos de persistencia de hipoacusia, luego de estape- dectomías satisfactorias en casos previamente diagnosticados como otoesclerosis.

Al examen físico, los pacientes afectados presentan nistagmus torsional evocado por sonidos o presión, fenómeno de Tullio, hipoacusia de conducción, reflejos acústicos intactos e hipersensibilidad a sonidos transmitidos por vía ósea. Con respecto a la evaluación audiológica se ha reportado que los pacientes pueden presentar hipoacusia de conducción moderada, incluso pudiendo simular curvas de otoesclerosis. Por otra parte, presentan una respuesta normal a los exámenes de discriminación y reflejos acústicos intactos ${ }^{6,7,15}$.

\section{DIAGNÓSTICO}

日 diagnóstico del SDCSS se basa en la historia clínica, el examen físico y los hallazgos radiológicos ${ }^{16}$. La sospecha clínica juega un papel fundamental en el diagnóstico de esta patología, ya que los síntomas y signos clásicos del cuadro pueden no ser evidentes al momento de la consulta.

Minor sugiere que la dirección torsional del nistagmus provocado por sonido y/o presión en el oído afectado puede orientarnos hacia el diagnóstico de SDCSS en un paciente que consulta por vértigo?.

La evaluación de estos pacientes debiera contemplar una audiometría, estudios de imágenes, evaluación vestibular y algunos autores sugieren el uso de potenciales miogénicos evocados vestibulares (VEMP) ${ }^{17,18}$.

Las imágenes juegan un rol fundamental en el diagnóstico del cuadro, ya que a pesar de que el SDCSS está definido fundamentalmente por el cuadro clínico, éstas permiten certificar la presencia de la dehiscencia en los canales semicirculares.

En general, la técnica de elección en este caso es la tomografía axial computada de hueso temporal. Los más recomendado son los cortes coronales, preferiblemente de $0,5 \mathrm{~mm}$ de grosor ${ }^{19}$. Aros autores han señalado de utilidad para el diagnóstico, aparte de los cortes coronales, cortes perpendiculares y paralelos al canal semicircular superior $^{20}$ (Figura 2). 


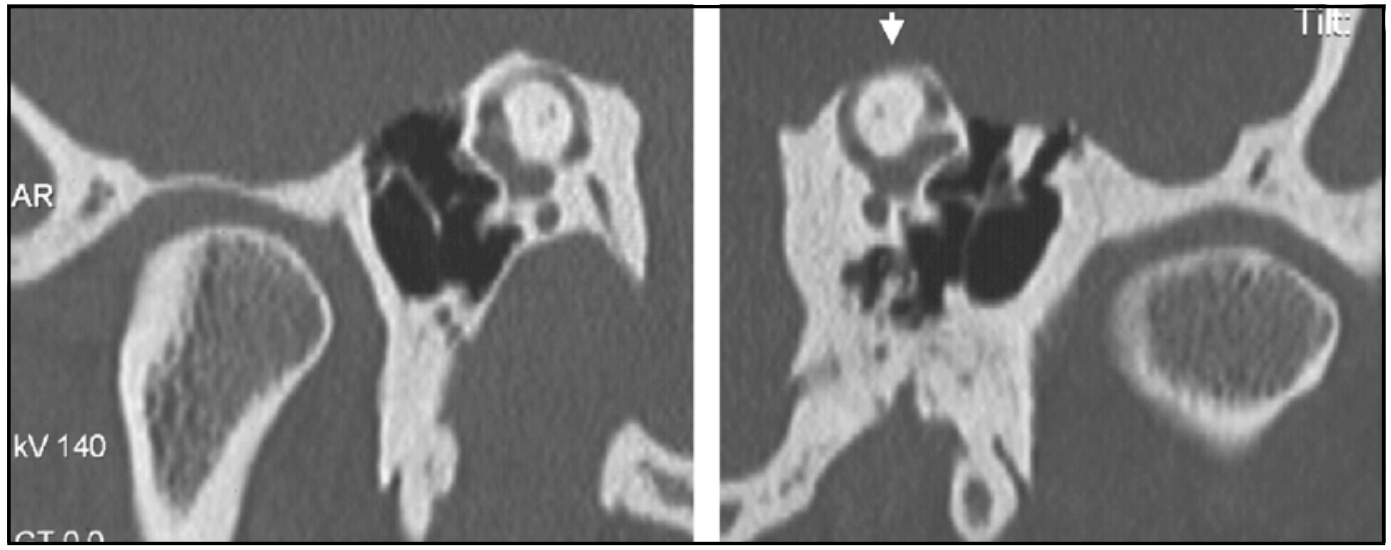

Figura 2. Tomografía axial computada en proyección de Poschl. Izquierda, examen normal. Derecha, dehiscencia del canal semicircular superior derecho (flecha). (Fotografía gentileza Dr. Steven Rauch, Massachussets Eye and Ear Infirmary, Harvard Medical School).

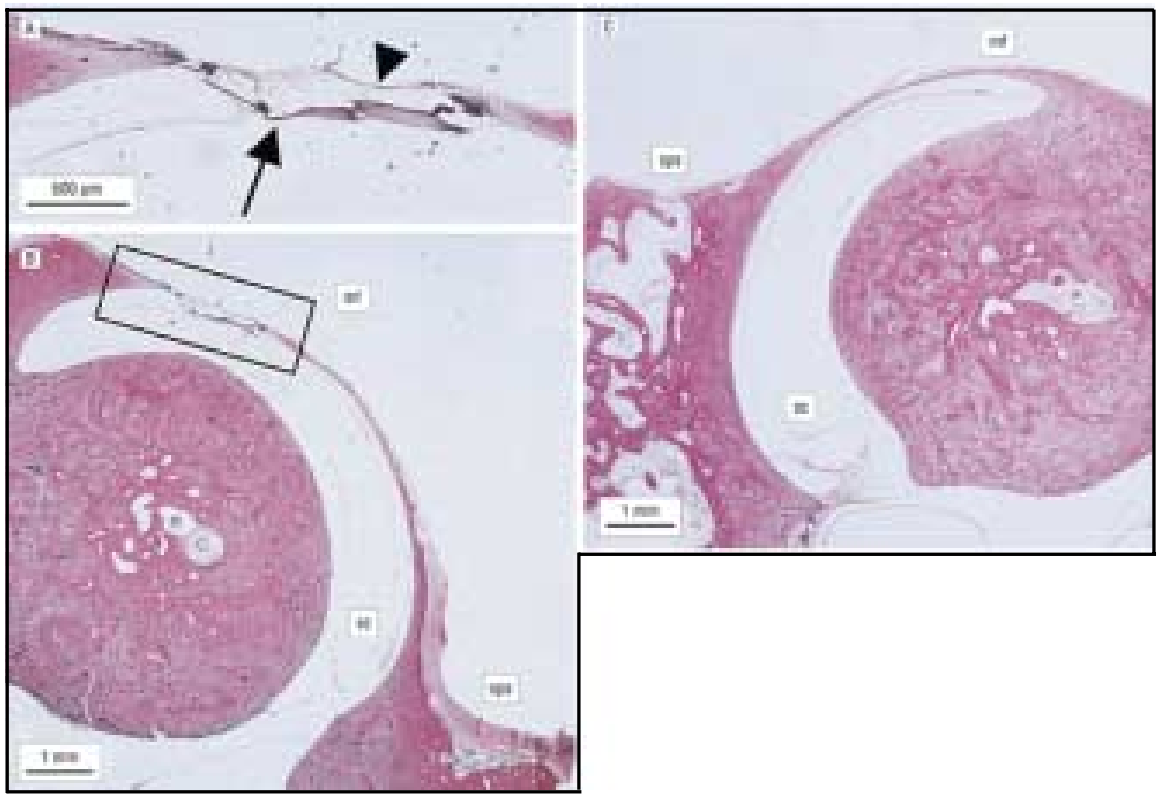

Figura 3. Corte histológico de hueso temporal en que se observa adelgazamiento de la pared de canal semicircular superior. Carey et al, $2000^{11}$.

Ara técnica, que ha sido reportada como de utilidad para el diagnóstico de este cuadro, son los potenciales miogénicos evocados vestibulares (VEMP). Los VEMP se basan en la sensibilidad acústica residual de sáculo. En esta técnica se registran los potenciales de relajación de latencia corta evocados por "clicks" y recogidos a través de electrodos en el músculo esternocleidomastoídeo 
ipsilateral mientras el músculo es sometido a contracción tónica $日$ umbral para evocar los potenciales miogénicos es menor en los pacientes que presentan SDCSS ${ }^{10,13,14}$.

Halmagyi y colaboradores evaluaron los movimientos oculares en respuesta a "clicks" de baja frecuencia en pacientes portadores de SDCSS y controles sanos. Observaron que los pacientes portadores de SDCSS presentaban movimientos oculares fácilmente reproducibles frente a la estimulación con "clicks" de baja frecuencia, y que la respuesta era diez veces mayor a la observada en sujetos sanos, por lo que plantean esta técnica como una alternativa válida de tamizaje para SDCSS en pacientes con vértigo y/o desequilibrio $^{21}$.

\section{TRATAMIENTO}

日 tratamiento del cuadro estará basado fundamentalmente en la clínica que presente el paciente y en el impacto en la calidad de vida de éste ${ }^{16}$.

En sus primeros reportes Minor señaló que, en la mayoría de los pacientes observados, el control del cuadro se pudo lograr sólo con evitar las maniobras desencadenantes de los síntomas ${ }^{6}$.

Sin embargo, en aquellos pacientes en que los síntomas sean incapacitantes, el tratamiento definitivo será la resolución quirúrgica de la dehiscencia. Minor describió el cierre de forma quirúrgica del defecto con una mezcla de fascia, hueso y goma de fibrina ${ }^{6,13}$. En los pacientes tratados observó una franca mejoría de la sintomatología vestibular y desaparición del fenómeno de Tullio.

En un trabajo reciente publicado por Mikulec y colaboradore $^{22}$ se analizaron los resultados clínicos de once pacientes con diagnóstico de SDCSS. Los pacientes fueron operados mediante craneotomía con abordaje a través de la fosa media, y después de identificar la dehiscencia, ésta era sellada con cera de hueso (una mezcla de cera de abejas e isopropil palmitato) hasta asegurar el cierre completo del defecto. La mejoría de la sintomatología se hacía evidente aproximadamente un mes después de la cirugía, sin embargo los autores reportan que algunos de los pacientes intervenidos presentaron hipoacusia sensorioneural moderada de frecuencias altas después de la cirugía como complicación.

Otras alternativas como fascia, goma de fibrina, injerto óseo y cemento de hidroxiapatita han sido usados también en la reparación del defecto, con diversos resultados ${ }^{19}$.

\section{CONCLUSIONES}

La dehiscencia del CSS se presenta como una entidad clínica recientemente descrita.

Las implicancias clínicas de este fenómeno son variadas. Oreemos que el SDCSS debe ser conside rado en pacientes que presenten vértigo producido por sonido y/o presión, ya que la identificación de una dehiscencia en el CSS implica una causa de vértigo potencialmente tratable.

\section{BIBLIOGRAFÍA}

1. NADO JB. Possitive Hennebert's sign in Ménière's disease. Arch Otolaryngol 1977; 103 : 524-30.

2. TuluIO P. Das Ohr und die Entstehung der Sprache und Schrift. Berlin, Germany: Urban \& Schwarzenberg; 1929.

3. HUIZINGA E On the sound reactions of Tullio. Acta Aolaryngol (Stockh) 1935; 22: 359-369.

4. HeNN⿴囗十T C. A new syndrome in hereditary syphilis of the labyrinth. Presse Med Belg Brux. 1911; 63 : 467.

5. MINOR LB. Laberynthine fistulae: pathobiology and management. Ourr Opin Otolaryngol Head Neck Surg 2003; 11: 340-6.

6. MINOR LB, SOLOMON D, ZENRIOH JS, ZEE DS. Sound- and/or pressure induces vertigo due to bone dehiscence of the superior semicircular canal. Arch Otolaryngol Head Neck Surg 1998; 124: 249-58.

7. MINOR LB. Superior canal dehiscence syndrome. Am J Ool 2000; 21: 9-19.

8. Hirvonen tP, CAREY JP, LIANG CJ, MinOR LB. 
Superior canal dehiscence: Mechanisms of pressure sensitivity in a chinchilla model. Arch Otolaryngol Head Neck Surg 2001; 127: 1331-6.

9. LeMPert T, Von Brevern M. Episodic vertigo. Ourr Opin Neurology 2005; 18: 5-9.

10. ROSOWSKI JJ, SONGR JE, NAKAJIMA HH, BRINSKO KM, MERCHANT SM. Cinical, experimental, and theoretical investigations of the effect of superior semicircular canal dehiscence hearing mechanisms. Otol Neurotol 2004; 25: 323-32.

11. CAREY JP, MinOR LB, NAGR GT. Dehiscence and thinning of bone overlying the superior semicircular canal in a temporal bone survey. Arch Aola ryngol Head Neck Surg. 2000; 126: 137-47.

12. WILLIAMSON RA, VRABEC JT, COKER NJ, SANDLIN M. Coronal computed tomography prevalence of superior semicircular canal dehiscence. Atolaryngol Head Neck Surg 2003; 129: 481-9.

13. MinOR LB, CAREY J, CREMER P, LUSTIG L, STREUB日 $S$. Dehiscence of bone overlying the superior canal as a cause of apparent conductive hearing loss. Otol Neurotol 2003; 24: 270-8.

14. SONGR JE, BRINSKO KM, ROSONSK JJ. Superior semicircular canal dehiscenceand boneconduction in chinchilla Abstracts of the twenty-sixth annual mid-winter research meeting Mt Royal, NJ: Association for research in otolaryngology 2003; 200.

15. MiKUleC A, MokenNa M, RaMSEY M, ROSOWSKI J,
Herrmann B, Rauch S, Ourtin H, Merchant S. Superior semicircular canal dehiscence presenting as conductive hearing loss without vertigo. Otol Neurotol 2004; 25: 121-9.

16. MontOJO J, HetzMANN T, RuBIO L. Dehiscencia del conducto semicircular superior: presentación de un caso y revisión bibliográfica. Acta Otorrinolaringol Esp 2005; 55: 171-5.

17. We gampOA M, COLBATCH J. Characteristics and clinical applications of vestibular-evoked myoge nic potencials. Neurology 2005; 64: 1682-88.

18. Wegampola M, Rosengren S, HaLmagy G, COLBATCH J. Vestibular activation by bone conducted sound. J Neurol Neurosurg Psychiatry 2003; 74: 771-8.

19. GIANOLI G Deficiency of the superior semicircular canal. Ourr Opin Otolaryngol Head Neck Surg 2001; 9: 336-41.

20. Mang A, Loenet L, Salaman D, Bigrow D. Sound- and pressure-induced vertigo associated with dehiscence of the roof of the superior semicircular canal. Am J Neuroradiol 1999; 20: 1973-75.

21. Halmagy GM, MoGarVie LA, YaVOR RA, TODD MJ. The clic-evoked vestíbulo-ocular reflex in superior semicircular canal dehiscence. Neurology 2003; 60: 1172-75.

22. MIKULECA, POED, MCKENNA. Operative management of superior semicircular canal dehiscence. Laryngoscope 2005; 115: 501-7.

Dirección: Dr. Jorge Caro Letelier

UDA Aorrinolaringología, Facultad de Medicina, Pontificia Universidad Católica de Chile

Email: jcarolet@med.puc.cl 